\title{
Short-range repression permits multiple enhancers to function autonomously within a complex promoter
}

\author{
Susan Gray, Paul Szymanski, and Michael Levine \\ Department of Biology, Center for Molecular Genetics, University of California at San Diego, La Jolla, California \\ 92093-0322 USA
}

\begin{abstract}
Transcriptional repressors play a key role in establishing localized patterns of gene expression in the early Drosophila embryo. Several different modes of repression have been implicated in previous studies, including competition and direct interference with the transcription complex. Here, we present evidence for "quenching," whereby activators and repressors co-occupy neighboring sites in a target promoter, but the repressor blocks the ability of the activator to contact the transcription complex. This study centers on a zinc finger repressor, snail (sna), which represses the expression of neuroectodermal regulatory genes in the presumptive mesoderm. We show that sna can mediate efficient repression when bound $50-100$ bp from upstream activator sites. Repression does not depend on proximity of sna-binding sites to the transcription initiation site. sna is not a dedicated repressor but, instead, appears to block disparate activators. We discuss the importance of quenching as a means of permitting separate enhancers to function autonomously within a complex promoter.
\end{abstract}

[Key Words: Transcriptional repressors; gene expression; Drosophila embryo; quenching; enhancers; complex promoter]

Received May 16, 1994; revised version accepted June 15, 1994.

Transcriptional repression is essential for the establishment of localized patterns of gene expression in the Drosophila embryo. Different modes of repression have been proposed, including competition, direct repression, and quenching (for review, see Levine and Manley 1989; see Fig. 1). Competition has been invoked to account for spatially restricted expression of segmentation genes in the early embryo. For example, even-skipped (eve) stripe 2 expression is regulated by a 480 -bp enhancer that contains six activator and six repressor sites (Small et al. 1992). Four of the six activator sites directly overlap four of the repressor sites. The core recognition sequence of the bicoid $(b c d)$ activator shares a 6 out of 10 match with the binding sites of the Krüppel $|K r|$ repressor (Driever and Nüsslein-Volhard 1989; Stanojevic et al. 1989; Treisman and Desplan 1989). These observations prompted the proposal that $K r$ defines the posterior stripe 2 border by blocking the binding of $b c d$ activators to overlapping sites (Small et al. 1991, 1992; Stanojevic et al. 1991). A similar mechanism has been suggested for the regulation of $K r$ (Hoch et al. 1992). Competition has also been implicated in cross-regulatory interactions among members of the steroid receptor superfamily le.g., Liu et al. 1993).

Localized patterns of zerknüllt (zen), decapentaplegic $(d p p)$, and tolloid (tld) expression appear to depend on a long-range mechanism of direct repression, perhaps akin to "silencing" (Ip et al. 1991; Huang et al. 1993; Jiang et al. 1993; Kirov et al. 1993, 1994). All three genes are repressed in ventral regions of early embryos in response to the dorsal (dl) gradient. Repression of the zen pattern is mediated by a distal promoter element, the ventral repression (VR) element, that contains high affinity $d l$ binding sites and closely linked corepressor sites. The VR is able to repress the ventral expression of a number of different heterologous promoters, including hunchback ( $h b$ ), Kr, and eve (Doyle et al. 1989; Ip et al. 1991; Jiang et al. 1993|. Ventral repression is observed even when the $d l$-corepressor-binding sites in the VR map several kilobases away from the closest activators in the heterologous promoters (Hoch et al. 1990; Ip et al. 1991). These observations suggest that the zen VR might act directly on the transcription complex. Certain repressors appear to interfere with the transcription complex when bound to proximal regions of target promoters. Among these are the Drosophila homeo domain proteins eve and engrailed [(en) Han and Manley 1993a,b], as well as the unliganded form of the mammalian thyroid hormone receptor (Fondell et al. 1993)

Here, we present evidence for repression through "quenching," whereby activators and repressors bind adjacent sites, but the repressors mask the ability of the activators to contact the transcription complex (see legend to Fig. 1). This study centers on the zinc finger re- 

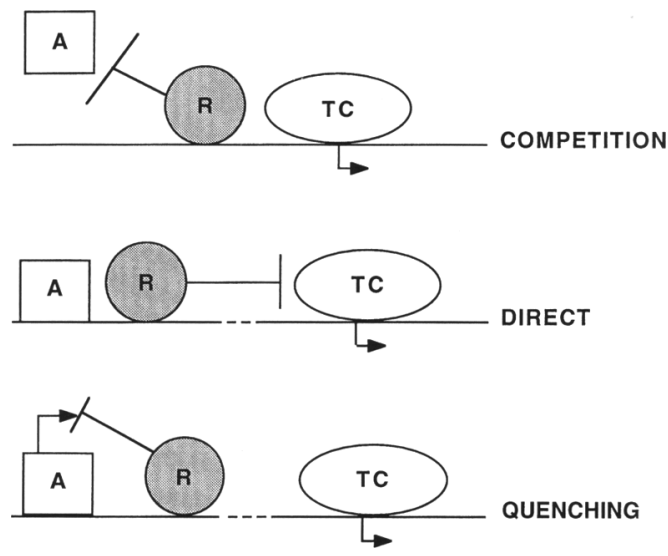

Figure 1. Modes of transcriptional repression. The diagrams summarize three different modes of repression. Competition involves the occlusion of activator sites by the binding of overlapping repressors. Direct repression involves interference with the formation or activity of the transcription complex (TC). Finally, according to a quenching mechanism, activator and repressor co-occupy neighboring sites, but the repressor masks the ability of the activator to contact the transcription complex.

pressor snail (sna), which is a key mesoderm determinant in the Drosophila embryo (Simpson 1983; Boulay et al. 1987). sna ${ }^{-}$mutants fail to gastrulate and lack mesoderm derivatives such as muscles and connective tissues (Simpson 1983; Alberga et al. 1991; Leptin 1991). sna defines the mesoderm/neuroectoderm boundary of the early embryo by repressing the expression of neuroectodermal regulatory genes in the presumptive mesoderm (Kosman et al. 1991; Leptin 1991). sna homologs have been implicated in the gastrulation of zebrafish (Hammerschmidt and Nüsslein-Volhard 1993; Thisse et al. 1993), frogs (Sargent and Bennett 1990), chicks (Nieto et al. 1994), and mice (Smith et al. 1992), so there is every expectation that its mechanism of action is evolutionarily conserved.

The rhomboid (rho) promoter is an early target of sna activity (Bier et al. 1990; Ip et al. 1992). It is expressed in two lateral stripes that coincide with the presumptive neuroectoderm of early embryos. rho stripes are regulated by the 300 -bp neuroectoderm enhancer element (NEE), which contains $d l$ - and HLH-binding sites that activate expression in both the lateral neuroectoderm and ventral mesoderm. Expression is excluded from the mesoderm by closely linked sna repressor sites. In sna ${ }^{-}$ mutants, the rho expression pattern is completely derepressed and spans both the lateral neuroectoderm and ventral mesoderm (Kosman et al. 1991).

We present evidence that sna-binding sites need not overlap activator sites to repress rho expression in the ventral mesoderm. Repression is observed when sna sites map 50 or $120 \mathrm{bp}$ from the closest $d l$ activator sites, although better repression occurs when the sna sites are $50 \mathrm{bp}$ from the activators. Efficient repression is not correlated with the proximity of the sna sites to the transcription initiation site. sna does not appear to be a "dedicated" repressor but, instead, can block the heterolo- gous $b c d$ activator. We discuss the importance of quenching as a means for permitting separate enhancers to function autonomously within a complex promoter.

\section{Results}

sna repressor sites need not overlap $\mathrm{dl} / \mathrm{HLH}$ activator sites

The rho NEE contains four high affinity $d l$-binding sites, five E boxes [helix-loop-helix (HLH) recognition sequences], and four sna repressor sites (see legend to Fig. 2). Previous studies have shown that mutations in the sna sites result in the ventral derepression of the rho pattern, so that there is equally intense staining in both the lateral neuroectoderm and ventral mesoderm (see Fig. 2C, D; Ip et al. 1992). To investigate the mechanism by which sna represses rho we have introduced synthetic sna-binding sites at various positions in and near a defective rho NEE lacking all four native sna repressor sites. The $\mathrm{s} 2$ recognition sequence was used in these experiments because other sna sites, such as $\mathrm{s} 3$ and $\mathrm{s} 4$, also include an E-box activator site (Ip et al. 1992; Kasai et al. 1992). Modified NEEs were attached to a lacZ reporter gene and then introduced into embryos via $P$ transformation. The resulting expression patterns were visualized by whole mount in situ hybridization using a $1 a c Z$ antisense RNA probe labeled with a digoxigenin-tagged UTP analog (Tautz and Pfeifle 1989; Jiang et al. 1991).

The first set of experiments involved the use of a 700bp DNA fragment that maps between -2.2 and $-1.5 \mathrm{~kb}$ upstream of the rho transcription start site. This fragment encompasses the entire 300-bp NEE as well as $\sim 200$ bp of flanking sequence on either side of the NEE. The flanking sequences lack high affinity $d l$, basic HLH (bHLH), and sna-binding sites (Ip et al. 1992). However, we cannot exclude the possibility that these sequences include low affinity sna repressor sites, because gastrulating embryos carrying the defective 700-bp NEE show slightly reduced levels of staining in the invaginating mesoderm (Fig. 2D).

Synthetic sna s2 sites were placed $\sim 150$ bp upstream and $\sim 120$ bp downstream of the closest $d l$ activators in the defective NEE (Fig. 2E,F). This modified NEE fusion gene, NEE-150, directs an abnormal pattern of expression in precellular embryos (Fig. 2E). Intense expression is detected in both the lateral neuroectoderm and ventral mesoderm, although there may be a slight attenuation in the levels of staining in the mesoderm. This pattern is quite similar to that observed for the unmodified NEE (cf. Fig. 2C). However, by the onset of gastrulation the NEE-150 fusion gene is slightly, but consistently, repressed in the mesoderm (Fig. 2F). This repression is more pronounced than the variably reduced ventral staining observed with the comparable NEE lacking synthetic sna sites (cf. Fig. 2D). It would appear that two sna sites, bracketing the defective NEE, can mediate slight repression when they map quite far $(120-150 \mathrm{bp})$ from the closest $d l$ activators. Repression may be more efficient in gastrulating embryos (Fig. 2, cf. F and E), as there 

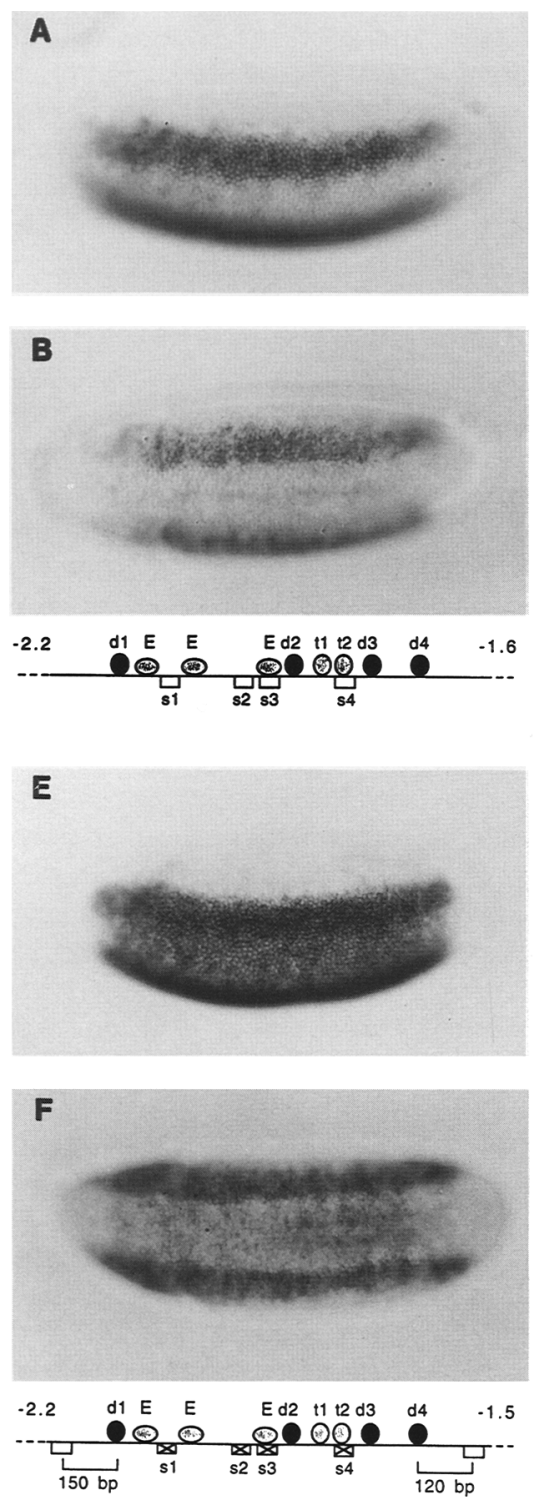
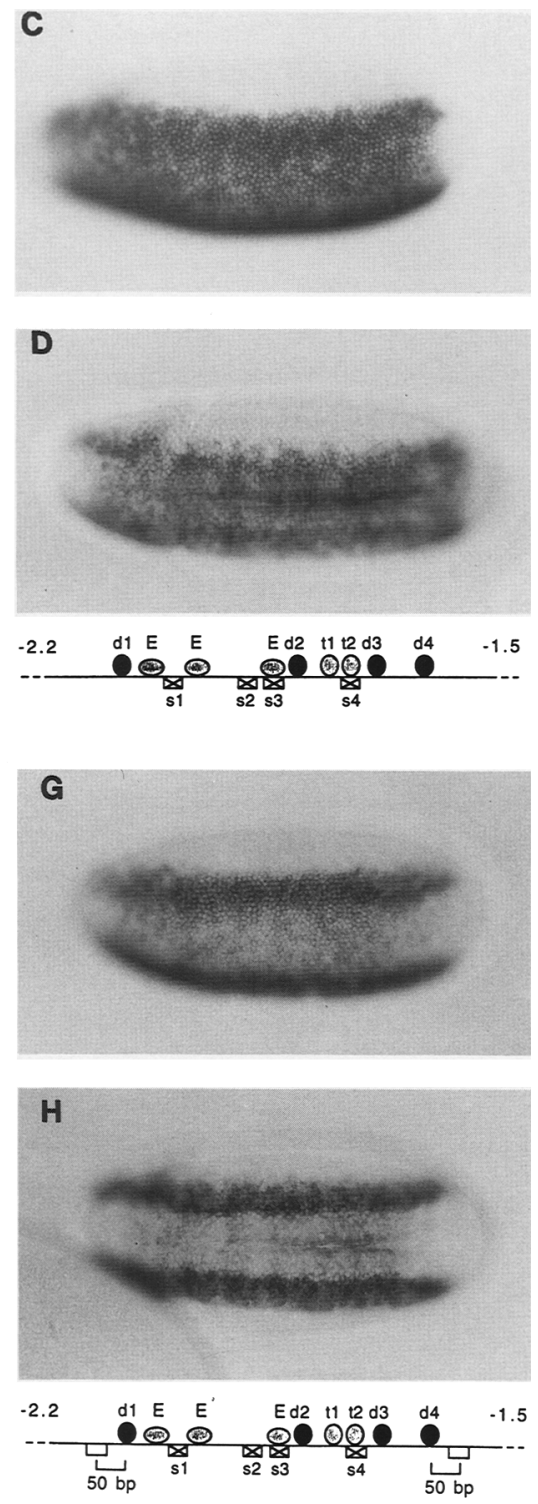

Figure 2. Uncoupling sna repressor sites and $d l / b H L H$ activator sites. Embryos were collected from $\mathrm{P}$ transformants and stained to visualize the expression of the lacZ reporter gene. Embryos are oriented with anterior to the left. $(A, C, E, G)$ Precellular embryos; $(B, D, F, H)$ gastrulating embryos. $(A, B)$ Expression of a wild-type $600-$ bp NEE-lacZ fusion gene. Staining is excluded from the mesoderm and restricted to lateral stripes in the neuroectoderm. $(C, D)$ Expression of a defective 700-bp NEE-lacZ fusion gene lacking all four snabinding sites. Staining is derepressed in the ventral mesoderm. $(E, F)$ Expression of a defective 700-bp NEE containing synthetic sna sites placed 150 bp upstream of the $d l \mathrm{dl}$ and $120 \mathrm{bp}$ downstream of the $\mathrm{d} 4$ site. There is a slight attenuation of the pattern in ventral regions early, which becomes more pronounced by the onset of gastrulation. $(G, H)$ Expression of the defective NEE with synthetic sna sites placed $50 \mathrm{bp}$ upstream of $\mathrm{dl}$ and $50 \mathrm{bp}$ downstream of $\mathrm{d} 4$. A nearly normal staining pattern is observed, with virtually complete repression in ventral regions. The diagrams (bottom) show the arrangement of factor-binding sites in the corresponding NEE. There are four $d l$ activator sites ( $\mathrm{d} l-\mathrm{d} 4$ ), five $\mathrm{E}$ boxes (two of these bind the twi HLH protein), and four sna sites. Boxed X's represent mutagenized binding sites; the synthetic s2 sna sites are denoted by open rectangles. is an increase in the levels of sna protein during cellularization (Alberga et al. 1991; Kosman et al. 1991; Leptin 1991).

The NEE-50 fusion gene, containing one sna s2 site 50 bp upstream and another $\mathrm{s} 2$ site $50 \mathrm{bp}$ downstream from the defective NEE directs an essentially normal pattern of expression (Fig. 2G,H). Lateral stripes are observed in precellular embryos, with only low levels of staining in the ventral mesoderm (Fig. 2, cf. G and A). Expression restricted to the neuroectoderm persists during cellularization and gastrulation (Fig. $2 \mathrm{H}$ ). These results indicate that sna-binding sites need not overlap $d l / \mathrm{bHLH}$ activator sites to mediate efficient ventral repression.

To investigate the stoichiometry of sna activator interactions, single $\mathrm{s} 2$ sites were placed in or near the defective NEE. A solo site located $50 \mathrm{bp}$ downstream of the NEE mediates efficient ventral repression (Fig. 3A), nearly comparable with that observed with the NEE-50 fusion gene containing two sna sites (Fig. 2G). A similar pattern is observed when the single site is located at its natural position within the NEE (Fig. 3B). Slightly less efficient repression is observed when the single site is placed 50 bp upstream of the NEE (data not shown). Thus, it would appear that a single sna protein can block multiple $d l$ and bHLH activators.

\section{sna can repress heterologous activators}

To determine whether sna is a dedicated repressor that blocks only $d l$ and/or bHLH activators, s2 sites were placed in both synthetic and natural promoter elements that are activated by the $b c d$ homeo domain protein. $b c d$ is distributed in a broad concentration gradient, with peak levels of protein in anterior regions and progressively lower levels in more posterior regions (Driever and Nüsslein-Volhard 1988). It is the primary activator of the 

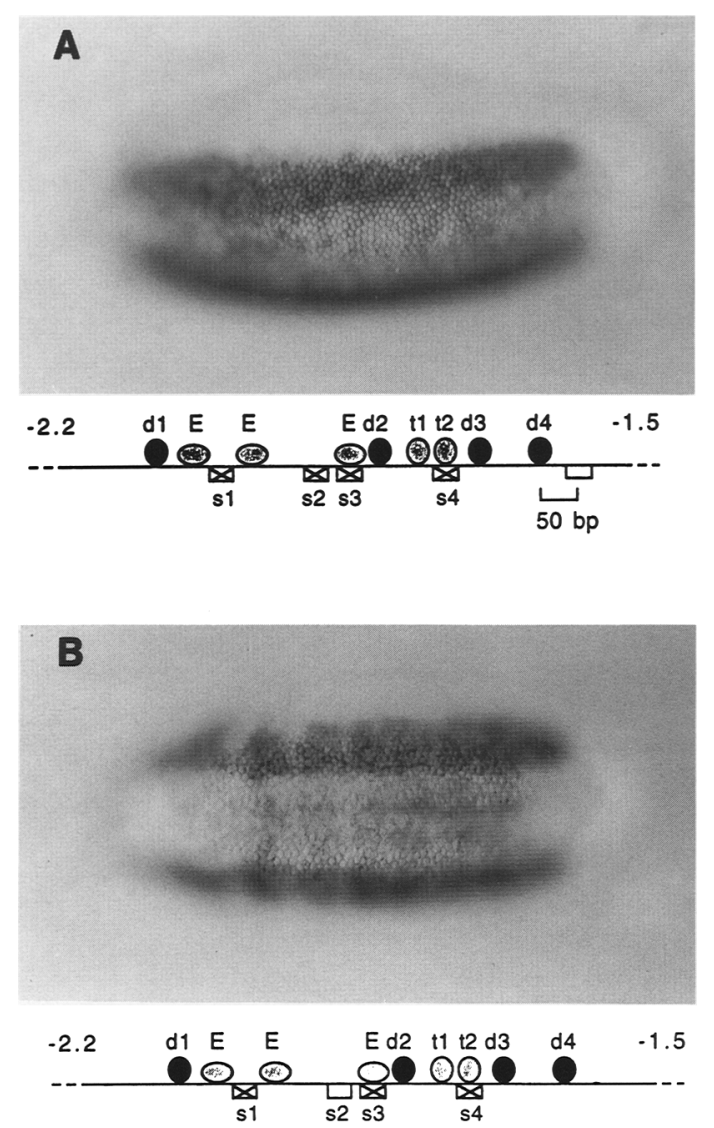

Figure 3. A single sna site can mediate repression. P-transformed embryos are presented as in Fig. 2. $\{A \mid$ Expression pattern of a precellular embryo containing the defective NEE with a single sna site placed $50 \mathrm{bp}$ downstream of $\mathrm{d} 4$. There is nearly normal repression in ventral regions. $(B)$ Expression pattern of a gastrulating embryo containing the defective NEE with a single sna site at the natural s2 position. An essentially normal tho pattern is observed. gap gene $h b$ and the pair-rule gene eve (Driever et al. 1989; Struhl et al. 1989; Stanojevic et al. 1991; Small et al. 1992). The first test of potential sna-bcd interactions involved the use of a synthetic promoter that contains three high affinity $b c d$ sites, three s2 sites, and three $d l$ sites (Fig. 4, see summary diagram below A and B). This synthetic element is a highly modified form of a 200-bp minimal rho NEE. It retains three of the native $d l$-binding sites $(\mathrm{d} 2, \mathrm{~d} 3$, and $\mathrm{d} 4)$, but the three $\mathrm{E}$ boxes were eliminated; two of the sites were replaced with the $b c d 1$ (b1) recognition sequence, which is a crucial activator of the eve stripe 2 enhancer (Small et al. 1992). In addition, the $\mathrm{s} 3$ site, which normally includes an $\mathrm{E}$ box, was converted into an $\mathrm{s} 2$ site. Finally, synthetic $\mathrm{s} 2$ and b1 sites were placed between the $\mathrm{d} 3$ and $\mathrm{d} 4 \mathrm{dl}$ sites.

The synthetic element directs a staining pattern that is consistent with synergistic activation by $d l$ and $b c d$, and repression by sna (Fig. 4A,B). Intense staining is observed in the anterior-most $20-30 \%$ of the embryo, where there are peak levels of $b c d$ protein. Progressively lower levels of staining exist in more posterior regions, coinciding with reduced amounts of $b c d$. Interestingly, the lateral limits of the pattern are broadest in anterior regions, but there is a progressive narrowing commensurate with reduced levels of $b c d$. There is no staining in the posterior-most regions in response to $d l$ alone. Staining is completely eliminated in the ventral mesoderm along the entire length of the embryo, suggesting that sna can repress activation by both $d l$ and bcd (Fig. 4B).

It is conceivable that $d l-s n a$ interactions are required for the efficient occupancy of the $s 2$ sites by the sna repressor. To determine whether sna can repress the $b c d$ activator in the absence of $d l$, all three $d l$ sites in the synthetic promoter element were mutagenized (Fig. 4, see summary diagram below $\mathrm{C}$ and $\mathrm{D}$ ). This results in the loss of the lateral stripes in the presumptive thorax and abdomen, providing additional evidence that these
Figure 4. sna can repress activation by $b c d$. Lateral $(A)$ and ventral $(B)$ views of a precellular embryo containing a modified 200-bp NEE with $d l-, b c d-$, and sna-binding sites. A combinatorial bcd-dl staining pattern is observed, with the progressive diminishment of lateral stripes in posterior regions. Staining is excluded from ventral regions coinciding with the limits of the sna repressor pattern. Lateral $(C)$ and ventral $(D)$ views of a precellular embryo containing the modified NEE shown in $A$ and $B$, except that the $d l$-binding sites were abolished by mutagenesis. Staining is restricted to anterior regions where there are peak levels of bcd but is excluded from ventral regions where there are high levels of the sna repressor. The diagrams below the embryos show the arrangement of factor-binding sites in the modified element, which includes three bcd sites, three $d l$ sites, and three sna sites.
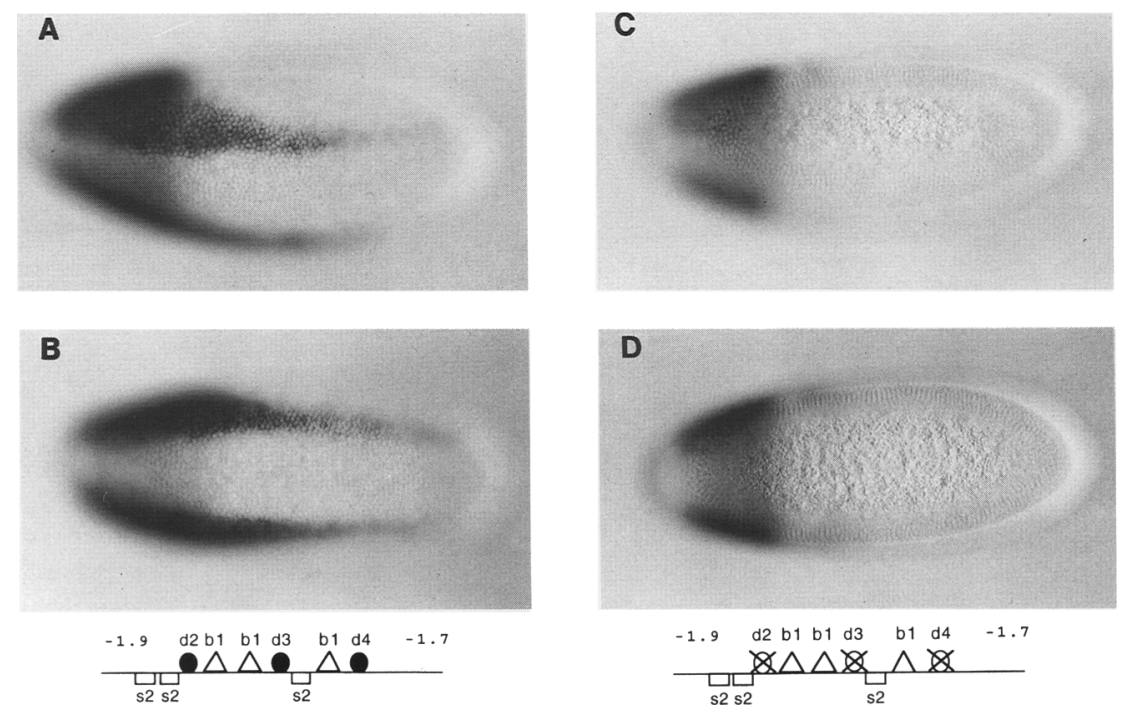
stripes depend on the combined action of $b c d$ and $d l$ (Fig. $4, \mathrm{cf}$. C and A). Staining is restricted to the anterior-most $20-30 \%$ of the embryo, where there are peak levels of $b c d$. Thus, activation of the modified element depends solely on $b c d$. Nonetheless, the staining pattern is excluded from the presumptive mesoderm in ventral regions (Fig. 4D), suggesting that sna is able to repress $b c d$ mediated activation in the absence of $d l$.

Another test of sna's ability to repress heterologous activators involved inserting $\mathrm{s} 2$ sites in the eve stripe 2 enhancer (Fig. 5, see summary diagram below B). Previous studies have shown that stripe 2 is activated by $b c d$ and $h b$, whereas the borders of the stripe are defined by the giant $(g t)$ and $K r$ repressors in anterior and posterior regions, respectively (Stanojevic et al. 1991; Small et al. 1992). The minimal 480-bp stripe 2 enhancer contains
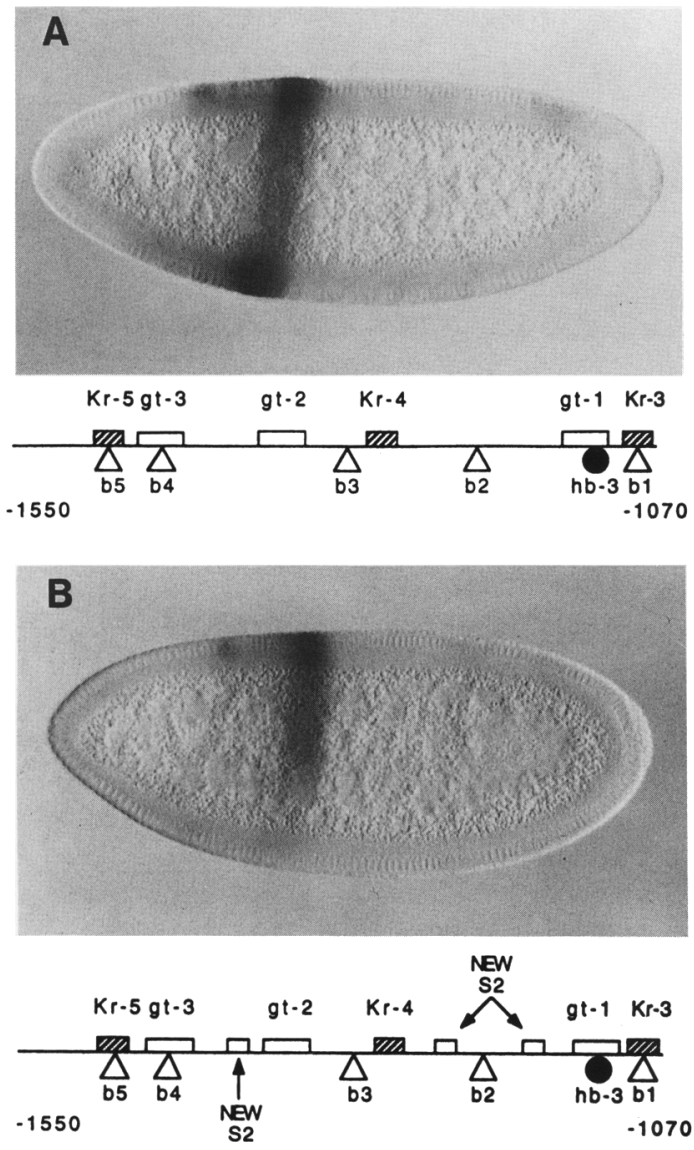

Figure 5. sna represses a heterologous enhancer. $(A)$ Precellular embryo expressing a lac $Z$ fusion gene containing the 480-bp eve stripe 2 enhancer. Uniformly intense staining is observed in dorsal and ventral regions. $(B)$ Precellular embryos expressing a modified stripe 2 enhancer containing three synthetic sna sites. Staining is excluded from ventral regions containing high levels of the sna repressor. The diagrams below the embryos show the wild-type and modified stripe 2 enhancers. There are six activator sites (five $b c d$ and one $h b$ ), and six repressor sites (three $K r$ and three $g t)$. The synthetic sna sites are indicated by open rectangles. six $b c d$ and $h b$ activator sites, as well as six $g t$ and $K r$ repressor sites (Fig. 5, see summary diagram below A). The stripe 2 enhancer is active in dorsal and ventral regions of early embryos (Fig. 5A). However, the introduction of three s2-binding sites within the stripe 2 enhancer results in the repression of the pattern in the ventral mesoderm (Fig. 5B). The domain of repression coincides with the limits of the sna expression pattern, suggesting that sna is able to repress the modified stripe 2 enhancer. It is unlikely that repression involves competitive binding to DNA, as the most closely linked s2 repressor site and $b c d$ activator site (Fig. 5, see summary diagram below B) map $\sim 40$ bp from each other (see Discussion).

\section{Short-range repression permits enhancer autonomy}

The preceding results are consistent with the notion that sna acts locally, within the limits of the enhancer, to mask the ability of activators to contact the transcription complex. An important implication of this mechanism is that a repressor bound to one enhancer should not interfere with another enhancer located elsewhere in the same promoter. To test this idea, composite promoters were examined that contain the eve stripe 2 enhancer attached to the rho NEE. These experiments involved the use of the 480-bp stripe 2 enhancer (Small et al. 1992) and either a 200 - or 152 -bp minimal NEE.

The first set of experiments involved placing the 152bp NEE upstream of the stripe 2 enhancer. This NEE contains two $d l$ sites, three E boxes, and three sna sites, including the $s 2$ sequence. The composite promoter directs an additive expression pattern that includes both eve stripe 2 and rho lateral stripes (Fig. 6A,B). There is no detectable diminishment in the ventral expression of stripe 2, suggesting that sna bound to the NEE does not interfere with stripe 2 activators (Fig. 6, A and B; cf. with Fig. 5B). The most closely linked sna repressor site in the NEE (s4) and activator site in the stripe 2 enhancer (bcd 5 ; b5) map $\sim 75$ bp away from each other, just beyond the range of efficient sna-mediated repression as judged by earlier experiments (e.g., Fig. 2). Although sna appears to exert little or no effect on stripe 2 expression, it would appear that the binding of $K r$ to the stripe 2 enhancer attenuates the expression of the rho lateral stripes (Fig. $6 \mathrm{~A}, \mathrm{~B})$. There is reduced expression in central regions, just posterior to the limit of stripe 2 . This coincides with the $K r$ expression domain (Rosenberg et al. 1985; see below). Very similar staining patterns, including attenuated expression of the rho stripes, were observed when the 152-bp NEE was placed in either a direct or inverted orientation upstream of the stripe 2 enhancer (Fig. 6, cf. A and $B$ ). The two orientations result in similar spacings of activators and repressors in the two enhancers.

$K r$ may repress over longer distances than sna, as the $K r$ and sna sites map a similar distance from the closest activator site in the neighboring enhancer (Fig. 6, see summary diagrams below A and B). Perhaps $K r$ exerts a stronger effect on the composite NEE/stripe 2 pattern, as the $K r$-binding sites map downstream of the NEE, close 

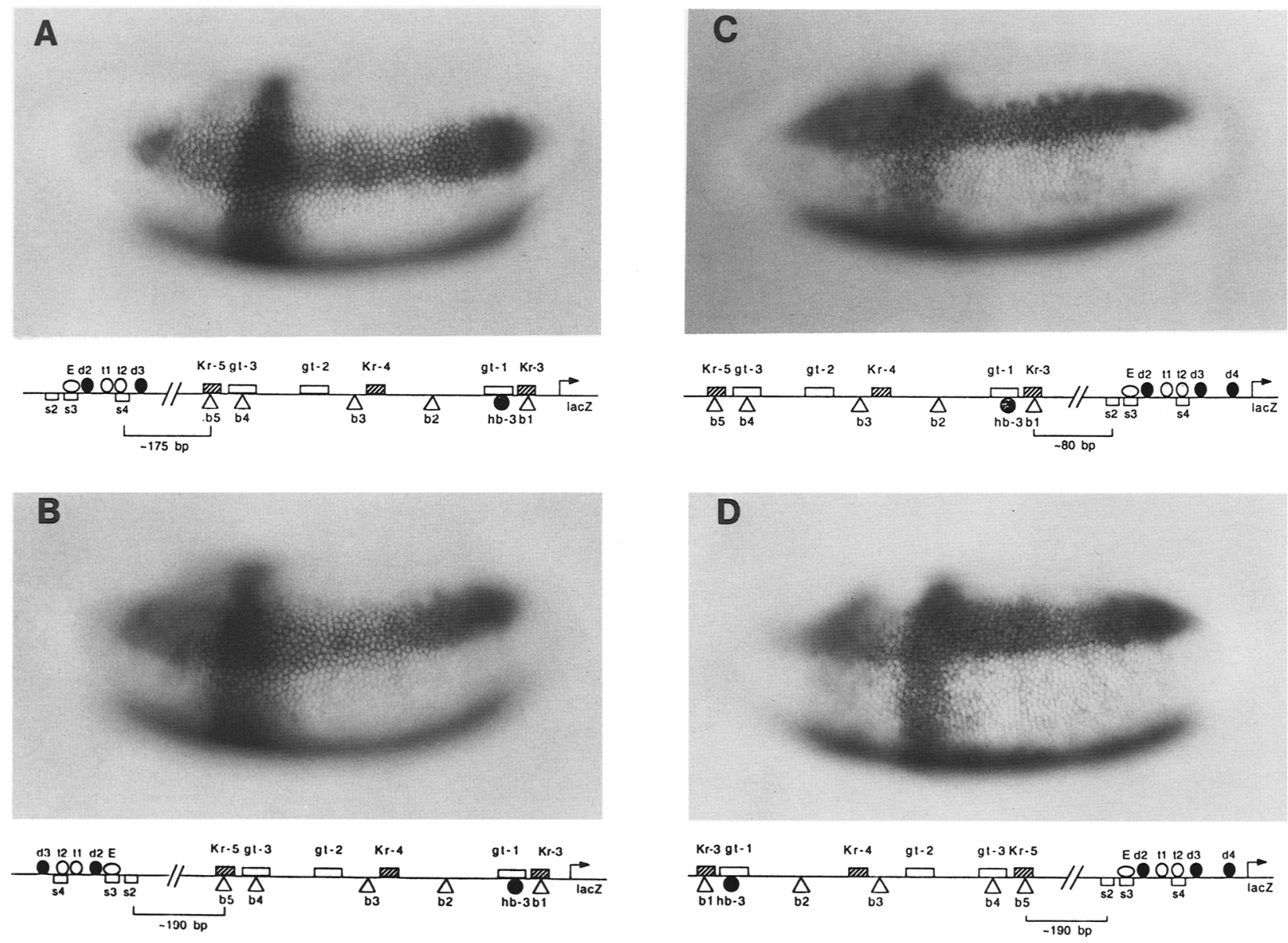

Figure 6. sna does not function as a silencer. P-transformed embryos express different NEE-stripe 2 fusion promoters. All embryos are at similar, precellular stages. (A) Expression pattern of an NEE-stripe 2 fusion promoter containing a 152-bp NEE in a direct $\left(5^{\prime} \rightarrow 3^{\prime}\right)$ orientation upstream of the 480 -bp eve stripe 2 enhancer. An additive staining pattern is observed, consisting of $r$ ho lateral stripes and eve stripe 2. There is a slight reduction in the staining levels in central regions coinciding with peak expression of the $K r$ repressor. $(B)$ Same as $A$ except that the fusion promoter contains the 152-bp NEE in an inverted $\left(3^{\prime} \rightarrow 5^{\prime}\right)$ orientation. The staining pattern is quite similar to that observed in $A$. $(C)$ Expression pattern of an eve stripe 2-NEE fusion promoter with the 480-bp eve enhancer in a direct $\left(5^{\prime} \rightarrow 3^{\prime}\right)$ orientation upstream of a 200-bp NEE. An additive staining pattern is observed, although there is a reduction in the ventral staining of eve stripe 2. (D) Same as $C$ except that the stripe 2 enhancer is in an inverted $\left(3^{\prime} \rightarrow 5^{\prime}\right)$ orientation. Ventral staining of the eve stripe is stronger than that observed in $C$. The diagrams below each embryo show the arrangement of factor-binding sites in the NEE and stripe 2 enhancers.

to TATA (within $20 \mathrm{bp}$ ). To determine whether the sna sites in the NEE can repress the stripe 2 enhancer in an orientation-dependent manner, fusion promoters were examined that contain a 200 -bp NEE placed downstream of the stripe 2 enhancer (Fig. 6C,D). Although sna sites now map relatively close to TATA (within $130 \mathrm{bp}$ ), stripe 2 expression extends through ventral regions where there are high levels of sna repressor. However, stripe 2 expression is weaker when the upstream stripe 2 enhancer is in a direct (Fig. 6C) versus an inverted (Fig. 6D) orientation relative to the transcriptional start site. The direct orientation places the s2 sna site within $80 \mathrm{bp}$ of the bl activator site, whereas the closest sna-bcd sites map $190 \mathrm{bp}$ in the inverted orientation. The former distance is within the range of sna-mediated repression /see
Fig. 2) and might result in the attenuated expression of stripe 2 (Fig. 6, cf. B and D).

Additional evidence that $K r$ can repress NEE activators was obtained by placing a single consensus $\mathrm{Kr}$-binding site on either side of a defective NEE lacking all four sna sites (Fig. 7, see summary diagram below B). This modified NEE is similar to the one shown in Figure 2 $\{\mathrm{G}, \mathrm{H}\}$, whereby synthetic sna s2 sites were placed $50 \mathrm{bp}$ upstream and $50 \mathrm{bp}$ downstream of the closest $d l$ activator site in the NEE. As shown previously, the defective NEE is expressed in both lateral and ventral regions of early embryos (Fig. 7A). However, the addition of the $K r$ sites causes a dramatic change in the staining pattern, such that expression is repressed in a central region coinciding with the $K r$ domain (Fig. $7 \mathrm{~B}$ ). There is repression 

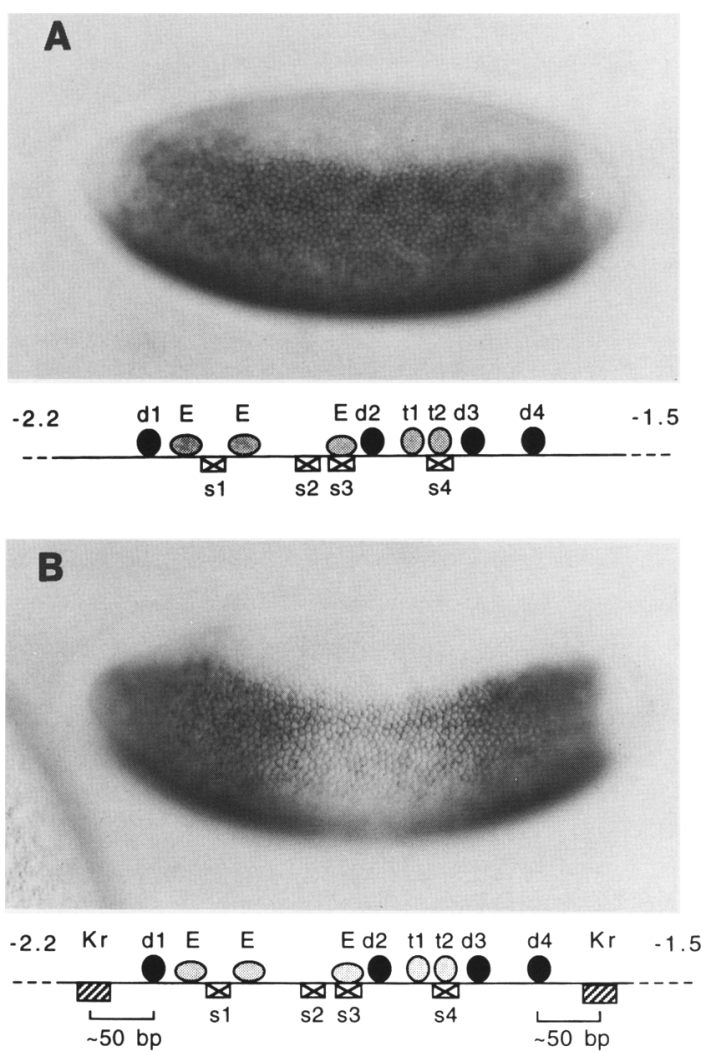

Figure 7. $K r$ represses a heterologous enhancer. $(A)$ Precellular embryo expressing a defective 700-bp NEE lacking all four sna sites (same as those shown in Fig. 2, C and D). A derepressed staining pattern is observed, with nearly uniform expression in lateral and ventral regions. $(B)$ Precellular embryo expressing the same NEE as in $A$, except that synthetic $K r$-binding sites were placed $50 \mathrm{bp}$ upstream of $\mathrm{d} 1$ and $50 \mathrm{bp}$ downstream of $\mathrm{d} 4$. Staining is repressed in central regions coinciding with the limits of the $K r$ pattern. The diagrams below the embryos show the arrangement of factor-binding sites in the wild-type and modified NEE.

of the pattern in both lateral and ventral regions, as the $K r$ repressor is equally distributed along the dorsoventral axis. This result indicates that $K r$ can efficiently repress heterologous activators in the context of a distinct enhancer. The simplest interpretation of this result is that $K r$, like sna, can mask the ability of closely linked activators to contact the transcription complex (see Discussion).

\section{Discussion}

Evidence is presented that sna functions through a shortrange mechanism of transcriptional repression. We favor the view that the repression involves quenching, whereby sna blocks the activities, but not binding, of nearby $d l$ and HLH transcriptional activators. There are numerous examples of short-range interactions among transcriptional activators, such as protein-protein contacts that facilitate cooperative occupancy of DNA-bind- ing sites (e.g., Jiang and Levine 1993). In principle, quenching could involve similar interactions between repressor and activator, although sna appears to function in a nonstoichiometric manner, in that one sna repressor site can efficiently block numerous activators. sna does not appear to be a dedicated repressor but, instead, may be able to block disparate activators. Quenching is a more flexible form of repression than competition because it does not require that activators and repressors recognize related DNA-binding sites. Short-range repression provides a basis for understanding how different enhancers can function autonomously within a complex promoter.

\section{Modes of repression}

Several lines of evidence suggest that sna does not repress rho by directly interfering with the transcription complex (direct repression; see Fig. 1). As sna sites are moved farther from a defective NEE but closer to TATA, there is a progressive loss in sna repression activity (Fig. 2 ). The NEE- 150 promoter is only poorly repressed by sna, even though one of the synthetic sna sites maps just 78 bp from TATA. In contrast, efficient repression is observed for the NEE-50 promoter, although the proximal sna site now maps $178 \mathrm{bp}$ from TATA. Analysis of stripe 2-NEE fusion promoters supports the view that proximity to TATA is not a critical determinant of repression efficiency. For example, sna sites in the NEE do not necessarily repress stripe 2 expression when placed near TATA. Attenuated expression is observed only when activators in the distal stripe 2 enhancer map within 100 bp of sna repressor sites in the NEE (see Fig. 6).

The native configuration of activator and repressor sites in the rho NEE suggested that sna might function through a competition mechanism of repression (see footnote to Fig. 1). One of the sna sites (s4) directly overlaps an $E$ box $(t 2)$, and gel mobility retardation assays indicate that there is mutually exclusive binding of the twist (twi) HLH activator and sna repressor to this sequence (Ip et al. 1992; R. Park and M. Levine, unpubl.). Two of the remaining sna sites map within $14 \mathrm{bp}$ of either an $\mathrm{E}$ box or $d l$ site. Despite this tight linkage, sna does not appear to function through competition.

Efficient repression of the rho pattern is observed when sna s2-binding sites are placed $50 \mathrm{bp}$ from the closest activator sites in a defective NEE (see Fig. 2). Weak, but consistent, repression is also observed when the s2 sites map as far as $120 \mathrm{bp}$ from the closest activator. Given these large distances, it seems unlikely that sna functions via competition. Gel mobility retardation assays indicate that mixtures of a truncated $d l$ protein $(d l$ 378 ) that is over half the size of the full-length protein and a glutathione $S$-transferase (GST)-sna fusion protein that is about the same size as full-length sna can cooccupy neighboring $d l$ - and sna-binding sites separated by just $6 \mathrm{bp}$ (J. Jiang and M. Levine, unpubl.). Similarly, the demonstration that $K r$ can repress the rho NEE over a distance of $50 \mathrm{bp}$ (Fig. 7) suggests that competition 
might not be important for the regulation of the eve stripe 2 enhancer.

\section{Mechanism of quenching}

It is conceivable that sna represses transcription by making local protein-protein contacts with closely linked activators. These interactions might be similar to the weak protein associations that permit certain activators to bind DNA in a cooperative fashion (e.g., Jiang and Levine 1993). According to this view a discrete domain within the sna protein might contact a neighboring activator so that its activation domain is unable to promote transcription. Although the nature of the sna-dl or sna-HLH interaction is still unclear, the results presented in this study indicate that sna blocks repression in a nonstoichiometric manner. There are several potential explanations for the ability of a single sna repressor to block many activators. Perhaps sna recruits a "repression complex" after it is bound to DNA, and this complex is able to interfere with multiple activators. This scenario would be similar to the recruitment of the tup1/ ssn 6 repression complex by the $\alpha 2$ homeo domain protein in yeast (Keleher et al. 1992). Alternatively, it is possible that multiple activators coordinate communication with the transcription machinery by recruiting an "adaptor" protein. A single repressor might be capable of disrupting a higher order conformation arising from interactions among activators and thereby block transcription. Ultimately, it will be interesting to determine whether sna shares any common features with another zinc finger repressor in Drosophila, Suppressor of Hairy Wing, which mediates directional repression over long distances (Geyer and Corces 1992).

sna does not appear to be a dedicated repressor that specifically blocks $d l$ and/or HLH activators. Several of the experiments presented in this study demonstrate that sna is able to block activation by bcd, even though none of the embryonic promoters characterized to date appear to be jointly regulated by these two regulatory factors. It is possible that sna can quench disparate activators that employ distinct mechanisms for contacting the transcription complex. Alternatively, it is possible that $d l$ and $b c d$ possess structurally related activation domains, even though they do not share any obvious sequence homologies. Future experiments will address this issue of specificity by analyzing chimeric $b c d$ or $d l$ proteins containing each of the well-characterized activation domains, including acidic, glutamine-rich, and proline-rich activators (for review, see Mitchell and Tjian 1989).

\section{Long-range vs. short-range repression}

Repression by quenching can account for the autonomous expression of different enhancers within a complex promoter. For example, the eve promoter contains separate enhancers for the regulation of individual stripes (Goto et al. 1989; Harding et al. 1989). The eve stripe 3 enhancer is expressed in central regions of the embryo where there are high levels of the $K r$ repressor. It evades repression by $K r$, as it lacks high affinity $K r$-binding sites (Stanojevic et al. 1989). Moreover, $K r$ bound to the stripe 2 enhancer fails to interfere with the expression of the stripe 3 enhancer because the two enhancers are separated by a $1.7-\mathrm{kb}$ spacer sequence (Small et al. 1993). According to a quenching mechanism, $K r$ acts only locally within the limits of the stripe 2 enhancer to block the $b c d$ and $h b$ activators. Previous studies have shown that $K r$ can attenuate stripe 3 expression when the spacer sequence is removed and the two enhancers are directly coupled (Small et al. 1993).

Fusion promoters containing both the NEE and stripe 2 enhancer direct an additive pattern of expression consisting of $r$ ho lateral stripes and eve stripe 2. This suggests that sna, like $K r$, also acts over short distances, within the limits of the NEE. Cross-enhancer interactions are observed only when repressors in one enhancer map near activators in the other. For example, there is reduced expression of stripe 2 when the upstream stripe 2 enhancer is placed in a direct orientation relative to the proximal NEE. In contrast, stronger expression is observed when the orientation of the stripe 2 enhancer is inverted (Fig. 6). The former fusion promoter contains a sna site within $80 \mathrm{bp}$ of a bcd activator site in the stripe 2 enhancer while the latter separates the two classes of binding sites by $>190 \mathrm{bp}$.

The passive, short-range effects of the sna and $K r$ repressors contrast with the results obtained with fusion promoters containing the zen VR silencer element. The VR is repressed in response to $d l$, and when attached to heterologous promoters, such as the eve stripe 2 enhancer, it eliminates their ventral expression (Ip et al. 1991; Jiang et al. 1993). Thus, long-range repressors such as those that bind the zen VR exert a dominant effect on the activities of other regulatory elements in a complex promoter, whereas the sna and $K r$ repressors act only over short distances and therefore do not interfere with activators present even in neighboring enhancers.

\section{Materials and methods}

\section{Plasmid constructions}

rho promoter elements were derived from a HindIII-SspI genomic DNA fragment spanning from $-2.2 \mathrm{~kb}$ to $+90 \mathrm{bp}$ relative to the putative rho transcription start site (Bier et al. 1990) in a pGEM $7 Z f+$ vector (Ip et al. 1992). The 700-bp rho NEE fragments were obtained by digestion with $\mathrm{BamHI}$ /located in the polylinker of the vector) at the $5^{\prime}$ end and Bsu36I at the $3^{\prime}$ end. The Bsu36I end was blunted with Klenow, and BamHI linkers were added to insert the element into the BamHI site of the P-transformation vector, C4PLZ, which contains the P-element promoter and transposase amino-terminal sequence fused inframe with the lacZ gene (Wharton et al. 1993). In the NEE-150 construct (see Fig. 2), the blunted Bsu36I end was unchanged, and the element was inserted into the $B a m H I$ site and blunted SphI site of the C4PLZ vector. A 600-bp NEE (Fig. 2A,B) was obtained by digestion with $B a m H I$ and $A$ vall, and blunted into the StuI site of the pWHL P-transformation vector, which contains a minimal hsp70 promoter (Driever et al. 1989). A 200-bp NEE (Fig. $6 \mathrm{C}, \mathrm{D}$ ) was obtained by digestion of the $600-\mathrm{bp} \mathrm{pWHL}$ 
fusion gene at the synthetic NotI $(-1892$ bp) and BamHI $(-1694 \mathrm{bp})$ sites. This 200 -bp fragment was inserted into the NotI and BamHI sites of C4PLZ. The 152-bp NEE (Fig. 6A,B) was obtained by digestion at the NotI site (-1892 bp) and synthetic SphI site $(-1740 \mathrm{bp})$. This fragment was inserted into the C4PLZ polylinker between NotI and SphI, and then excised from this vector to make the 152-bp NEE-stripe 2 fusion promoter (see below).

The eve stripe 2 enhancer is $480 \mathrm{bp}$ in length and maps between $-1.55 \mathrm{~kb}$ and $-1.07 \mathrm{~kb}$ upstream of the eve transcription start site. This was attached to a basal eve-lac $Z$ fusion gene inserted into the CaSpeR injection vector (Small et al. 1992). The 152-bp NEE-stripe 2 fusion promoter was prepared by removing the 152-bp NEE from C4PLZ by digestion with HindIII (which cuts in the $3^{\prime}$ polylinker). EcoRI linkers were attached to this fragment after blunting with Klenow. It was subsequently inserted into the EcoRI site located $5^{\prime}$ of the 480-bp stripe 2 enhancer in the CaSpeR vector. The 200-bp stripe 2-NEE fusion promoter was prepared by inserting the 480-bp stripe 2 enhancer into the EcoRI site of the C4PLZ vector containing the 200-bp NEE (see above).

\section{$P$ transformation and whole-mount in situ hybridization}

$\mathrm{P}$ transposons containing various $l a c Z$ reporter genes were introduced into the Drosophila germ line using standard methods (Spradling and Rubin 1982). Multiple independent transformed lines were examined for each construct. lacZ reporter gene expression was visualized by whole-mount in situ hybridization using a digoxigenin-UTP-labeled $l a c Z$ antisense RNA probe (Tautz and Pfeifle 1989; Jiang et al. 1991).

\section{Site-directed mutagenesis}

The pGEM7Zf + plasmid containing the HindIII-SspI fragment from the rho 5 '-flanking region, and the pBluescript $\mathrm{SK}+$ plasmid containing the 480-bp BstEII-FspI fragment spanning the eve stripe 2 enhancer, were used to transform the CJ236 strain of Escherichia coli to prepare uracil-containing single-stranded DNA templates. Mutagenesis was done with the Muta-Gene kit (Bio-Rad, NY) by annealing the templates with various mutagenic oligonucleotides, as suggested by the manufacturer. The in vitro-synthesized double-stranded DNA was transformed into the DH5 $\alpha$ strain of $E$. coli. The identities of the mutagenized templates were confirmed by nucleotide sequence analysis. Synthetic sna s2 sites (CAGCAAGGTG) were created at the following positions in the rho promoter (all numbers refer to distance from the putative transcription start site): -2133 to $-2124 \mathrm{bp} ;-2021$ to $-2012 \mathrm{bp} ;-1839$ to $-1830 \mathrm{bp} ;-1748$ to $-1739 \mathrm{bp} ;-1658$ to $-1649 \mathrm{bp} ;-1595$ to $-1586 \mathrm{bp}$. Synthetic s2 sites were created at the following positions within the eve stripe 2 enhancer: -1395 to $-1386 \mathrm{bp} ;-1244$ to $-1235 \mathrm{bp}$; -1162 to -1153 bp. Synthetic $K r 1$ sites (AAAACGGGTTAAGC) were created at the following positions in the rho promoter region: -2023 to $-2010 \mathrm{bp} ;-1652$ to $-1639 \mathrm{bp}$. Synthetic $b c d 1$-binding sites (GGATTAGGG) were created at the following positions within the rho promoter: -1794 to -1786 bp; -1780 to $-1772 b_{p} ;-1732$ to $-1724 \mathrm{bp}$. Mutations that disrupt native sna and $d l$-binding sites in the NEE are described by Ip et al. (1992).

\section{Acknowledgments}

We thank Stephen Small and Tony Ip for help and encouragement. This work was supported by grants from the National Institutes of Health (GM 46638 and GM 34431).
The publication costs of this article were defrayed in part by payment of page charges. This article must therefore be hereby marked "advertisement" in accordance with 18 USC section 1734 solely to indicate this fact.

\section{References}

Alberga, A., J.L. Boulay, E. Kempe, C. Dennefeld, and M. Haenlin. 1991. The snail gene required for mesoderm formation in Drosophila is expressed dynamically in derivatives of all three germ layers. Development 111: 983-992.

Bier, E., L.Y. Jan, and Y.N. Jan. 1990. rhomboid, a gene required for dorsoventral axis establishment and peripheral nervous system development in Drosophila melanogaster. Genes \& Dev. 4: 190-203.

Boulay, J.L., C. Dennefeld, and A. Alberga. 1987. The Drosophila developmental gene snail encodes a protein with nucleic acid binding fingers. Nature 330: 395-398.

Doyle, H.J., R. Kraut, and M. Levine. 1989. Spatial regulation of zerknüllt: A dorsal-ventral patterning gene in Drosophila. Genes \& Dev. 3: 1515-1533.

Driever, W. and C. Nüsslein-Volhard. 1988. A gradient of bicoid protein in Drosophila embryos. Cell 54: 83-93.

- 1989. The bicoid protein is a positive regulator of hunchback transcription in the early Drosophila embryo. Nature 337: 138-143.

Driever, W., G. Thoma, and C. Nüsslein-Volhard. 1989. Determination of spatial domains of zygotic gene expression in the Drosophila embryo by the affinity of binding sites for the bicoid morphogen. Nature 340: 363-367.

Fondell, J.D., A.L. Roy, and R.G. Roeder. 1993. Unliganded thyroid hormone receptor inhibits formation of a functional preinitiation complex: Implications for active repression. Genes \& Dev. 7: 1400-1410.

Geyer, P.K. and V.G. Corces. 1992. DNA position-specific repression of transcription by a Drosophila zinc finger protein. Genes \& Dev. 6: 1865-1873.

Goto, T., P.M. Macdonald, and T. Maniatis. 1989. Early and late periodic patterns of even-skipped expression are controlled by distinct regulatory elements that respond to different spatial cues. Cell 57: 413-422.

Hammerschmidt, M. and C. Nüsslein-Volhard. 1993. The expression of a zebrafish gene homologous to Drosophila snail suggests a conserved function in invertebrate and vertebrate gastrulation. Development 119: 1107-1118.

Han, K. and J. Manley. 1993a. Transcriptional repression by the Drosophila even-skipped protein: Definition of a minimal repression domain. Genes \& Dev. 7: 491-503.

- 1993b. Functional domains of the Drosophila Engrailed protein. EMBO /. 12: 2723-2733.

Harding, K., T. Hoey, R. Warrior, and M. Levine. 1989. Autoregulatory and gap gene response elements of the evenskipped promoter of Drosophila. EMBO J. 8: 1205-1212.

Hoch, M., C. Schroder, E. Seifert, and H. Jäckle. 1990. cis-acting control elements for Krüppel expression in the Drosophila embryo. EMBO I. 9: 2587-2595.

Hoch, M., N. Gerwin, H. Taubert, and H. Jäckle. 1992. Competition for overlapping sites in the regulatory region of the Drosophila gene Krüppel. Science 256: 94-97.

Huang, J.D., D.H. Schwyter, J.M. Shirokawa, and A.J. Courey. 1993. The interplay between multiple enhancer and silencer elements defines the pattern of decapentaplegic expression. Genes \& Dev. 7: 694-704.

Ip, Y.T., R. Kraut, M. Levine, and C.A. Rushlow. 1991. The dorsal morphogen is a sequence-specific DNA-binding pro- 
tein that interacts with a long-range repression element in Drosophila. Cell 64: 439-446.

Ip, Y.T., R.E. Park, D. Kosman, E. Bier, and M. Levine. 1992. The dorsal gradient morphogen regulates stripes of rhomboid expression in the presumptive neuroectoderm of the Drosophila embryo. Genes \& Dev. 6: 1728-1739.

Jiang, J. and M. Levine. 1993. Binding affinities and cooperative interactions with bHLH activators delimit threshold responses to the dorsal gradient morphogen. Cell 5: 741-752.

Jiang, J., D. Kosman, Y.T. Ip, and M. Levine. 1991. The dorsal morphogen gradient regulates the mesoderm determinant twist in early Drosophila embryos. Genes \& Dev. 5: 18811891.

Jiang, J., H. Cai, Q. Zhou, and M. Levine. 1993. Conversion of a dorsal-dependent silencer into an enhancer: Evidence for dorsal corepressors. EMBO J. 12: 3201-3209.

Kasai, Y., J.R. Nambu, P.M. Lieberman, and S.T. Crews. 1992. Dorsal-ventral pattern in Drosophila: DNA binding of snail protein to the single-minded gene. Proc. Natl. Acad. Sci. 89: 3414-3418.

Keleher, C.A., M.J. Redd, J. Schultz, M. Carlson, and A.D. Johnson. 1992. Ssn6-Tup1 is a general repressor of transcription in yeast. Cell 68: 709-719.

Kirov, N., L. Zhelnin, J. Shah, and C. Rushlow. 1993. Conversion of a silencer into an enhancer: Evidence for a co-repressor in dorsal-mediated repression in Drosophila. EMBO $\mathrm{I}$. 12: 3193-3199.

Kirov, N., S. Childs, M. O'Connor, and C. Rushlow. 1994. The Drosophila dorsal morphogen represses the tolloid gene by interacting with a silencer element. Mol. Cell. Biol. 14: 713722.

Kosman, D., Y.T. Ip, M. Levine, and K. Arora. 1991. Establishment of the mesoderm-neuroectoderm boundary in the Drosophila embryo. Science 254: 118-122.

Leptin, M. 1991. twist and snail as positive and negative regulators during Drosophila mesoderm development. Genes \& Dev. 5: 1568-1576.

Levine, M. and J.L. Manley. 1989. Transcriptional repression of eukaryotic promoters. Cell 59: 405-408.

Liu, Y., N. Yang, and C.T. Teng. 1993. COUP-TF acts as a competitive repressor for estrogen receptor-mediated activation of the mouse lactoferrin gene. Mol. Cell. Biol. 13: 18361846.

Mitchell, P.J. and R. Tjian. 1989. Transcriptional regulation in mammalian cells by sequence-specific DNA binding proteins. Science 245: 371-378.

Nieto, M.A., M.G. Sargent, D.G. Wilkinson, and J. Cooke. 1994. Control of cell behavior during vertebrate development by slug, a zinc finger gene. Science 264: 835-839.

Rosenberg, U.B., A. Preiss, E. Seifert, H. Jäckle, and D.C. Knipple. 1985. Production of phenocopies by Kruppel antisense RNA injection into Drosophila embryos. Nature 313: 703706.

Sargent, M.G. and M.F. Bennett. 1990. Identification in Xenopus of a structural homologue of the Drosophila gene snail. Development 109: 967-973.

Simpson, P. 1983. Maternal-zygotic gene interactions during the formation of the dorsoventral pattern in Drosophila embryos. Genetics 105: 615-632.

Small, S., R. Kraut, T. Hoey, R. Warrior, and M. Levine. 1991. Transcriptional regulation of a pair-rule stripe in Drosophila. Genes \& Dev. 5: 827-839.

Small, S., A. Blair, and M. Levine. 1992. Regulation of evenskipped stripe 2 in the Drosophila embryo. EMBO J. 11: 4047-4057.

Small, S., D.N. Arnosti, and M. Levine. 1993. Spacing ensures autonomous expression of different stripe enhancers in the even-skipped promoter. Development 119: 767-772.

Smith, D.E., F. Franco del Amo, and T. Gridley. 1992. Isolation of sna, a mouse gene homologous to the Drosophila genes snail and escargot: Its expression pattern suggests multiple roles during postimplantation development. Development 116: 1033-1039.

Spradling, A.C. and G.M. Rubin. 1982. Transposition of cloned P elements into Drosophila germline chromosomes. Science 218: 341-347.

Stanojevic, D., T. Hoey, and M. Levine. 1989. Sequence-specific DNA-binding activities of the gap proteins encoded by hunchback and Krüppel in Drosophila. Nature 341: 331335.

Stanojevic, D., S. Small, and M. Levine. 1991. Regulation of a segmentation stripe by overlapping activators and repressors in the Drosophila embryo. Science 254: 1385-1387.

Struhl, G., K. Struhl, and P.M. Macdonald. 1989. The gradient morphogen bicoid is a concentration-dependent transcriptional activator. Cell 57: 1259-1273.

Tautz, D. and C. Pfeifle. 1989. A nonradioactive in situ hybridization method for the localization of specific RNAs in Drosophila embryos reveals a translational control of the segmentation gene hunchback. Chromosoma 98: 81-85.

Thisse, C., B. Thisse, T.F. Schilling, and J.H. Postlethwait. 1993. Structure of the zebrafish snail1 gene and its expression in wild type, spadetail and no tail mutant embryos. Development 119: 1203-1215.

Treisman, J. and C. Desplan. 1989. The products of the Drosophila gap genes hunchback and Krüppel bind to the hunchback promoters. Nature 341: 335-337.

Wharton, K.A. Jr. and S.T. Crews. 1993. CNS midline enhancers of the Drosophila slit and Toll genes. Mech. Dev. 40: 141154. 


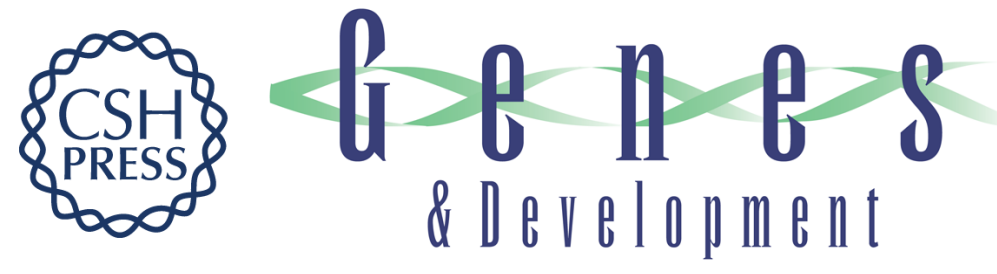

\section{Short-range repression permits multiple enhancers to function autonomously within a complex promoter.}

S Gray, P Szymanski and M Levine

Genes Dev. 1994, 8:

Access the most recent version at doi:10.1101/gad.8.15.1829

References This article cites 47 articles, 25 of which can be accessed free at:

http://genesdev.cshlp.org/content/8/15/1829.full.html\#ref-list-1

License

Email Alerting

Service

Receive free email alerts when new articles cite this article - sign up in the box at the top right corner of the article or click here.

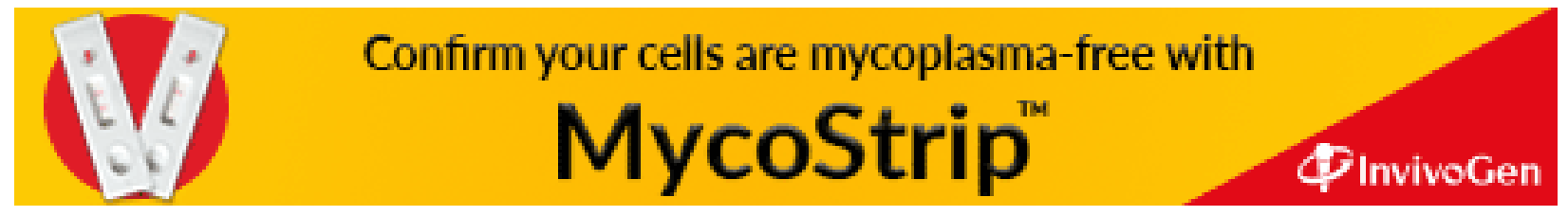

